\title{
Rastreamento do risco de perda funcional: uma estratégia fundamental para a organização da Rede de Atenção ao Idoso
}

\author{
Screening the risk for functional loss: \\ a basic strategy for organizing the elderly health care network
}

\author{
Célia Pereira Caldas ${ }^{1}$ \\ Renato Peixoto Veras ${ }^{1}$ \\ Luciana Branco da Motta ${ }^{1}$ \\ Kenio Costa de Lima ${ }^{2}$ \\ Cynthia Beatriz Silveira Kisse ${ }^{1}$ \\ Cláudia Valéria Moreno Trocado ${ }^{1}$ \\ Ana Carolina Lima Cavaletti Guerra ${ }^{1}$
}

\footnotetext{
${ }^{1}$ Universidade Aberta da Terceira Idade (UnATI), Centro Biomédico,

Universidade do Estado do

Rio de Janeiro. R. São

Francisco Xavier 524/bloco

F/10150, Maracanã.

20.599-900 Rio de Janeiro

RJ Brasil.

celpcaldas@hotmail.com

${ }^{2}$ Departamento de

Odontologia, Centro de

Ciências da Saúde,

Universidade Federal do

Rio Grande do Norte.
}

\begin{abstract}
From a critical analysis of experiments in the use of screening tools for functional loss in the elderly, it was observed that the main motivation to develop and implement a screening instrument is to improve health care for the frail elderly or those at risk of functional loss and repeated hospital admissions. The instruments are preferably short questionnaires with no more than 16 selfreported questions. The main intervention performed after screening was monitoring the elderly by a specialized nursing service that, when necessary, referring them to the medical service. The results of this approach included the reduction of functional loss, hospital admissions, deaths, lower heal th expenses and greater consumer satisfaction. Key words Screening, Elderly, Risk, Frailty
\end{abstract}

Resumo A partir de uma análise crítica sobre experiências com a utilização de instrumentos de rastreio de perda funcional em idosos, observou-se que a principal motivação para desenvolver e aplicar esta técnica é aperfeiçoar a assistência à saúde para os frágeis ou com risco de perda funcional e internações repetidas. Os instrumentos são preferencialmente questionários com até 16 perguntas autoadministráveis. A principal intervenção executada após o rastreio foi o acompanhamento do idoso por um serviço de enfermagem especializado que, quando necessário, referenciou-o ao serviço médico. Esta abordagem resultou na redução da perda funcional, do número de admissões hospitalares, do número de óbitos, redução de gastos com saúde e ainda uma maior satisfação do usuário.

Palavras-chave Rastreamento, Idosos, Risco, Fragilidade 


\section{Introdução}

Nos últimos anos o envelhecimento da população gera discussões envolvendo as necessidades e as peculiaridades dos idosos como usuários dos sistemas de saúde, seja este público ou privado. É intensa a preocupação com a manutenção da qualidade da assistência à saúde dos idosos sem perder o controle dos custos para manter os tratamentos necessários ${ }^{1-4}$. As altas taxas de hospitalização de idosos exemplifica o impacto do envelhecimento da população brasileira no setor de saúde e representam um grande desafio para os sistemas de saúde, sob o risco de ter seus leitos bloqueados sem oferecer a assistência necessária às demandas desse grupo 5 .

Uma boa assistência à saúde é inerente à confiança e ao vínculo do paciente com o profissional construído no acolhimento e em todo processo do cuidar ${ }^{6-9}$. Este gerenciamento deveria ser iniciado na porta de entrada e acompanhar o paciente durante toda participação do usuário no sistema de saúde ${ }^{2}$.

$\mathrm{Na}$ atual abordagem da prestação de serviços à saúde, há uma fragmentação do cuidado, multiplicando-se consultas, exames e outros procedimentos ${ }^{10}$. Neste cenário, a assistência à saúde não se apresenta como uma prestação de serviço segura para o idoso e seus familiares. Consequente a esta ineficiência, os setores público e privado estão sobrecarregados, gerando atendimentos por vezes desnecessários, o que implica em alto custo para os sistemas de saúde ${ }^{10,11}$.

A fragilidade tem sido apontada como um indicador de risco para a morte ou para a perda da funcionalidade em idosos, sendo utilizado como orientador no gerenciamento da saúde ${ }^{12-14}$.

$\mathrm{O}$ uso de ferramentas para rastreio de fragilidade e perda da capacidade funcional é utilizado em diversos serviços e sistemas de saúde, com o objetivo de identificar precocemente os idosos frágeis e direcionar a atuação geriátrica, prevenindo internações repetidas, aumentando a qualidade de vida e em alguns casos evitando a morte precoce ${ }^{13,15}$.

A palavra fragilidade indica um estado de instabilidade, com risco de perda funcional resultante de eventos por vezes previsíveis?. Tanto os instrumentos de rastreio de perda da capacidade funcional quanto os de risco de fragilidade, consideram em sua construção, características do estado biológico, cognitivo, funcional e circunstâncias sociais ${ }^{14,16-19}$.

Assim, os instrumentos de rastreio utilizados para identificar idosos com maior risco de inter- nação hospitalar avaliam alguns elementos das atividades instrumentais e básicas de vida diária, e características presentes em idosos frágeis, como o número de comorbidades, quantidade de medicação prescrita, perda de peso não intencional, dentre outros.

Em um estudo de revisão sistemática publicada em 2012 sobre ferramentas para rastreamento de fragilidade em idosos na atenção primária, os autores questionam em qual cenário deve ser aplicado ${ }^{20}$. Como o instrumento é aplicado, há as questões de quem responde às perguntas e a partir de qual idade deve-se iniciar o rastreio para risco de fragilização, considerando-se o sistema de saúde e o nível socioeconômico e cultural da população envolvida.

O objetivo deste trabalho foi analisar criticamente as experiências com a utilização de instrumentos de rastreio de perda funcional em idosos.

\section{Metodologia}

Foi realizada uma revisão crítica da literatura utilizando descritores e palavras chaves nos idiomas inglês ou português quando necessário.

As bases de dados pesquisadas foram a PubMed (NCBI); Web of science; The Cochrane Library; Lilacs, IBECS, MedLine, CIDSaúde, MedCarib, WHOLIS, PAHO e LIS através da Biblioteca Virtual em Saúde (BVS); e Scientific Electronic Library Online (Scielo).

Foram utilizados os descritores: screening, assessment, risk, disability, frail, performance, aged, aged 80 e over; e as palavras aging e tools. A busca na base de dado Scielo foi realizada utilizando os descritores e palavras já citadas, nos idiomas inglês e português, sendo acrescentados os termos evaluation, elderly, hospitalization, fragilização e hospitalização. Os descritores e as palavras foram combinados a fim de encontrar o maior número de publicações possíveis nas bases de dados.

O processo de identificação foi realizado por seis pesquisadores. O processo de seleção foi realizado por três pesquisadores e a leitura integral dos trabalhos foi realizada individualmente por outros cinco, de modo que cada artigo foi lido por 2 pessoas, utilizando um terceiro revisor para a síntese dos dados.

Utilizando descritores relacionados com rastreio de perda funcional em idosos, foram identificadas 71.074 publicações em 12 bases de dados bibliográficos. Após a eliminação das duplicidades, e de uma pré-seleção baseada nos critérios de 
inclusão e exclusão, foram selecionados 98 experiências de rastreamento de risco de perda funcional ou fragilidade em idosos.

Uma segunda seleção foi realizada nas 98 publicações para incluir somente trabalhos que utilizassem instrumento breve, de fácil aplicação, não necessitando de profissionais especializados para a execução. Nesta etapa foram lidos os resumos de todos os 98 artigos resultando na seleção de 25 publicações.

Outras 10 publicações foram selecionadas a partir da identificação de um artigo de revisão sistemática sobre ferramentas de rastreio de fragilidade no cuidado à saúde primária, publicado em março de 2012 por Pialoux et al. ${ }^{20}$.

Após a leitura e análise dos 35 artigos que tratam da utilização de instrumentos para rastreio de perda funcional e fragilidade em idosos, extraiu-se o cenário em que eles foram aplicados.

Foram adicionadas mais 2 publicações com a finalidade de complementar informações que não constavam no artigo selecionado pela busca bibliográfica. A partir deste acréscimo executouse a leitura integral de 37 publicações.

\section{Resultados}

$\mathrm{Na}$ revisão sistemática publicada por Pialoux et al. ${ }^{20}$, os autores selecionaram dez instrumentos de rastreio e avaliaram suas propriedades psicométricas em relação a uma avaliação geriátrica ou às medidas da Avaliação Geriátrica Ampla (AGA). Eles sugerem dois instrumentos potencialmente adequados, o Tilburg Frailty Indicators e o Share-FI. Segundo os autores estes instrumentos ainda requerem validação em estudos maiores na atenção primária e com critério de qualidade maior.

O Tilburg Frailty Indicators (TFI) ${ }^{21}$ é um questionário composto por 15 questões objetivas autorreferidas, validado na cidade de Roosendaal, Holanda, em duas amostras de idosos com idade de 75 anos ou mais $(\mathrm{n}=245 ; \mathrm{n}=234)$, no período de novembro de 2007 a junho de 2008. O resultado fornece escore que varia de 0 a 15 pontos e quanto maior a pontuação maior a fragilidade. O tempo necessário para preenchimento são 14 minutos e foi entregue por entrevistador treinado na casa do idoso.

Os participantes das duas amostras responderam a uma escala de qualidade de vida, o WHOQOL-BREF (World Health Organization Quality of Life), de modo que cada item do TFI foi correlacionado com o item correspondente na escala de qualidade de vida. Assim, de acordo com o estudo, o resultado do TFI possui forte correlação com a qualidade de vida, em particular os componentes psicológicos e sociais da fragilidade, fortalecendo a evidência da definição integral da fragilidade.

A adaptação transcultural do Tilburg Frailty Indicator para a população brasileira foi realizada por Santiago et al. ${ }^{22}$ e as 15 questões referem-se à sensação de boa saúde, perda de peso não intencional, problemas causados pela dificuldade em caminhar, equilíbrio, audição, visão, força de preensão manual, cansaço e memória. Incluem ainda a sensação de tristeza, nervosismo ou ansiedade, resiliência, moradia, suporte e apoio social.

O instrumento SHARE-FI (Survey of Health, Ageing and Retirement in Europe $)^{23}$ é um instrumento de 21 questões, mais um teste de força de preensão de mão, desenvolvido a partir de quatro domínios: exaustão, perda de peso, fraqueza muscular e baixa atividade física.

O objetivo do estudo foi desenvolver um instrumento válido e simples para medir o nível de fragilidade em pessoas com 50 anos de idade ou mais, na Europa. Foi desenvolvido a partir do processo de avaliação em doze países europeus (Áustria, Alemanha, Suécia, Holanda, Espanha, Itália, frança, Dinamarca, Grécia, Suíça, Bélgica e Israel) em duas etapas, compreendendo os anos de 2004 a 2006. Profissionais de saúde não médicos avaliaram individualmente numa primeira etapa 17.304 mulheres e 13.811 homens. Numa segunda etapa, 11.384 mulheres e 9.163 homens. Esta idade foi estabelecida porque segundo os autores, não há um consenso internacional que fixe uma idade como ponto de corte para definição de fragilidade. Há sim um reconhecimento que a fragilidade atua mais sobre a idade biológica que a cronológica dos indivíduos ${ }^{24}$.

Ao final desenvolveu-se um cálculo para a fragilidade em mulheres e outro para os homens, assim como um escore (Dfactor) de fragilidade para cada gênero.

Nesta pesquisa, 66,9\% das mulheres foram identificadas como não frágeis; $25,8 \%$ pré-frágeis e 7,3\% frágeis. O Odds Ratio para mortalidade entre as mulheres consideradas pré-frágeis foi de $2,1(1,4-3,0)$ e entre as frágeis, foi 4,8(3,1-7,4).

Quanto aos homens, $82,3 \%$ foram identificados como não frágeis, $14,6 \%$ pré- frágeis e 3,1\% frágeis. O Odds Ratio para mortalidade entre eles foi 3,0 (2,3-4) nos identificados como pré-frágeis e 6,9 (4,7-10,2) para os homens frágeis.

Os autores concluíram que o instrumento SHARE-FI pode ser utilizado por profissionais 
de saúde médicos e não médicos, em toda comunidade Europeia, pela fácil aplicabilidade e acessibilidade via web ${ }^{23}$.

Um estudo publicado por Amici et al. ${ }^{25}$ descreve a criação e a aplicação de um instrumento composto por 49 questões autorreferidas para rastreio de fragilidade em pessoas idosas, baseados na Marigliano-Cacciafesta Polypathological Scale (MCPS).

O estudo foi desenvolvido e aplicado em um centro de reabilitação da Universidade de Roma "La Sapienza". As questões compreendem patologias neurológicas, alterações cognitivas e humor, audição, visão, patologias cardíacas, renais, venosas, respiratórias, gástricas, metabólicas, alteração no estado nutricional, mobilidade e presença de tumores.

O instrumento foi entregue no dia da consulta médica, antes de fazer avaliação multidimensional geriátrica. Foi permitida a ajuda parcial ou total do cuidador no preenchimento do questionário.

Em 1996, Moore e Siu ${ }^{26}$ publicaram um artigo onde tratam do desenvolvimento e aplicação de um instrumento que denominaram Screening $\mathrm{Pa}$ ckage. A partir da revisão da literatura, desenvolveram o instrumento com 16 itens e o aplicaram em uma população de 109 novos pacientes atendidos no ambulatório de medicina geriátrica da universidade da Califórnia, em um estudo transversal.

$\mathrm{O}$ instrumento foi aplicado por profissionais não médicos previamente treinados. Para cada aplicação, o tempo variou de 8 a 12 minutos. Identifica problemas que classificam o idoso de risco, como desnutrição, perda de peso, perda visual, auditiva ou cognitiva, incontinência urinária, depressão, perda da capacidade física e da mobilidade.

Os autores concluíram que o pacote de triagem para avaliar problemas comuns em idosos é simples e possível de ser aplicado por profissionais não médicos durante a visita ao consultório, devido ao tamanho e à facilidade de execução ${ }^{26}$.

Em 1980, um grupo de pesquisadores ${ }^{27} \mathrm{pu}$ blicou um artigo onde descrevem o desenvolvimento de um questionário postal de rastreio para identificação de pacientes idosos que necessitavam de avaliação específica.

O questionário consiste em nove perguntas que representam aspectos da saúde e bem estar com respostas dicotômicas ( $\operatorname{sim} /$ não). Indagam, por exemplo, se o idoso possui algum parente para ajudá-lo em caso de necessidade, se os problemas de saúde o impedem de sair de casa, lhe causam preocupação ou dificuldades, se possui problemas de visão ou audição e se buscou atendimento hospitalar no ano anterior.

O estudo foi realizado na cidade de Glasgow, Escócia, em uma amostra randomizada de 102 pacientes com idade de 70 anos ou mais, usuários de um centro de saúde voltado ao atendimento das classes sociais 3,4 e 5 . Essas pessoas receberam uma carta contendo o questionário e a orientação para respondê-la no prazo de duas semanas. Uma resposta positiva (sim) ou não responder a carta e reenvia-la no prazo recomendado, já seria indicador de estado de risco, indicando que o sujeito precisaria de avaliação e acompanhamento especial. O acompanhamento foi realizado por dois visitadores de saúde e um enfermeiro distrital.

Das 102 cartas enviadas, 83 (81\%) foram respondidas. Dos 67 idosos identificados com risco, $61(91 \%)$ realmente necessitavam da avaliação e acompanhamento. Os autores concluíram que a utilização do instrumento de triagem poderia reduzir em um quinto a carga de trabalho de um programa de avaliação.

$\mathrm{Na} \mathrm{Holanda}^{28}$, foi aplicado um questionário para identificar perda funcional e fatores que interferem na qualidade de vida dos idosos. O Groninguen Activity Restriction Scale (GARS), um questionário de 18 itens com 4 possibilidades de respostas autoadministradas foi aplicado a 4.792 pessoas com idade de 57 anos ou mais, residentes da região norte da Holanda. As entrevistas foram realizadas por telefone ou visita domiciliar por mulheres de meia idade bem treinadas. $\mathrm{O}$ instrumento em questão não identificou o risco de perda funcional, mas a presença dela entre os idosos. Segundo os autores, o GARS pode ser útil para resumir o nível de incapacidade da população.

Em 1996, um grupo de pesquisadores apresentou um estudo onde descrevem o desenvolvimento de um questionário postal com a capacidade de prever o declínio funcional de pessoas idosas da comunidade ${ }^{29}$.

O questionário contendo 21 perguntas foi enviado a uma amostra de 842 idosos com idade igual ou superior a 75 anos, residentes da cidade de Sherbrooke (Quebec, Canadá). Esses idosos foram visitados em seus domicílios por uma enfermeira treinada em dois momentos, um mês após enviarem seus questionários e após um ano de seguimento. Ela realizou uma entrevista e aplicou uma avaliação funcional de autonomia.

$\mathrm{O}$ risco de declínio funcional foi associado à idade e a mais 14 itens do questionário. Após uma regressão logística, apresentaram 6 itens capazes de predizer o risco de declínio funcional, constituindo o Sherbrooke Postal Questionnaire. 
O questionário Sherbrooke foi aplicado em dois bairros de Londres, nos períodos de 19992002, com a finalidade de prever a utilização de serviços de emergência, providenciando assistência social e de saúde para reduzir os riscos. O questionário de 6 questões autoadministradas, foi enviado por correspondência para 2307 indivíduos com mais de 75 anos. Os autores concluíram que o questionário é um bom preditor para uso de serviços de emergência e hospitalar ${ }^{30}$.

Em estudo realizado na área de Limburg a Utrecht, Holanda ${ }^{31}$, foram aplicados os questionários autoadministrados Groningen Frailty Indicator (GFI), Tilburg Frailty Indicator (TFI) e Sherbrook Postal Questionnare (SPQ) com a finalidade de verificar o valor preditivo positivo destes três instrumentos de rastreio de fragilidade em idosos. Para tal foram comparados com outro instrumento que avalia incapacidade nas atividades de vida diária e com o GARS (Groningen Activity and Restriction Scale). Os questionários foram entregues por dois médicos a 687 indivíduos com mais de 70 anos de idade residentes nas áreas mencionadas. Destes, 532 responderam ao questionário e enviaram por correio. Após 1 ano de seguimento, 514 questionários foram reenviados por correspondência aos respondentes. Os autores concluem que o TFI e o SPQ são melhores preditores para a internação que o GFI e que os indivíduos identificados como frágeis tem 2 vezes mais chance de serem internados no período de 1 ano.

No Chile ${ }^{32}$, o Sherbrook Postal Questionnare foi aplicado em 2116 idosos com idade de 65 a 99 anos, na comuna de Lo Espejo, Santiago, Chile. Foi obtida a classificação de risco por idade e sexo. A partir dos resultados, os autores sugerem que sejam desenvolvidas ações coordenadas na atenção primária, secundária e terciária para a prevenção, o tratamento e a reabilitação.

A ferramenta PRISMA- $7^{33}$ foi desenvolvida em Quebec, Canadá e é utilizada no projeto PRISMA, administrada por profissionais de saúde na atenção domiciliar, informação de saúde por contato telefônico, assistência à pacientes agudos (por exemplo, setor de emergência), e também por agências de voluntários para avaliar incapacidade em pessoas idosas. Consiste em 7 perguntas objetivas com resposta dicotômica ( $\operatorname{sim} /$ não). O ponto de corte utilizado é 3 "sim” com uma sensibilidade de 78,2\%, rastreando o idoso que deve ser melhor avaliado.

O questionário contem perguntas referentes à idade, sexo, limitação funcional devido a problemas de saúde, necessidade de ajuda para atividades diárias, ter alguém para ajudar em caso de necessidade e uso de órtese para auxiliar na locomoção.

Em Quebec foi realizada uma pesquisa com o objetivo de medir o impacto do modelo PRISMA na saúde, satisfação e empoderamento de pessoas idosas, a utilização dos serviços e os encargos para seu cuidador principal informal ${ }^{34}$. Foram avaliadas 1501 pessoas entre 2001 e 2005, com idade de 75 anos ou mais na primeira etapa do estudo e 77 anos ou mais na segunda. Eles foram previamente identificados pelo Sherbrooke Postal Questionnaire tendo risco de perda funcional. Foi também aplicado o questionário PRISMA-7 e SMAF (Functional Autonomy Measurement System).

Na comparação entre PRISMA-7 e Sherbrooke Postal Questionnaire, os autores relatam que não houve diferença significativa quanto ao declínio funcional entre os grupos. Ambos detectaram que $30 \%$ dos participantes foram hospitalizados durante o primeiro ano da pesquisa.

Os autores relatam ainda que devido à predição de risco de perda funcional e integração ao sistema PRISMA, ocorreu uma melhor utilização dos serviços de saúde, diminuição da procura ao serviço de emergência, hospitalização e institucionalização. Neste estudo, 45\% dos idosos recusaram-se a participar do modelo integrado de assistência.

O questionário BRIGHT (Brief Risk Identification of Geriatric Health Tool ${ }^{35}$ foi desenvolvido para identificar pessoas idosas da comunidade com perda da capacidade funcional. Foi enviado por correspondência para ser preenchido e devolvido. Posteriormente os idosos foram avaliados por uma enfermeira gerontóloga treinada, através de uma avaliação geriátrica abrangente, o Minimum Dataset for Home Care (MDSHC). Foram avaliados 120 idosos com idade igual ou superior a 75 anos, residentes em Auckland, Nova Zelândia.

O questionário possui 11 itens, com possibilidade de resposta dicotômica e ponto de corte em 3 respostas afirmativas para identificação do risco de perda funcional, orientando o acompanhamento especial pelo serviço de saúde.

Nos EUA, um questionário desenvolvido para avaliar síndromes geriátricas foi utilizado para direcionar o atendimento especializado a 150 idosos com 65 anos ou mais, frequentadores de igrejas, centros sociais para idosos, lar de idosos aposentados de baixa renda e de um centro de reabilitação para idosos ${ }^{36}$. Os autores relatam que o instrumento contendo 35 questões com duração de 11 minutos, é bom preditor de hospitalização 
e que houve redução da mortalidade entre esses idosos após intervenção.

Em 1998, outros pesquisadores desenvolveram um questionário autoadministrado, considerando a fragilidade como dificuldade em mais de um dos domínios cognitivo, sensorial, físico ou nutricional ${ }^{37}$. O questionário elaborado possui 16 itens referentes aos quatro domínios. Foi aplicado em 48 idosos entre 63 e 90 anos, correlacionado com a avaliação geriátrica ampla. Diante das disparidades étnico-sociais e número pequeno de entrevistados, os autores concluíram não poder utilizar este instrumento para avaliar a fragilidade, mas sugerem que pode ser utilizado para prever a perda funcional.

Durante o Swiss Interdiciplinary Longitudinal Study on the Oldest (SWILO-O) ${ }^{38}$, um questionário contendo 17 itens foi aplicado em 1.225 octogenários no período de 1995 a 1999 em áreas urbanas e rurais da Suíça. Os itens compreendem os domínios: sensorial, mobilidade, dor física, memória e energia. A avaliação foi realizada por profissional treinado. A dependência funcional em atividades de vida diária dos idosos foi acompanhada no período de 12 a 18 meses após a avaliação. Os cinco domínios foram considerados preditores do início de dependência funcional e para a mortalidade.

$\mathrm{Na}$ presença de deficiência entre 2 e 3 domínios, o risco de desenvolver dependência funcional quase dobra. Quando a pessoa apresenta deficiência em 4 ou mais domínios, o risco de ter dependência é de 8,05\%. Quanto ao risco de morte, 2 ou 3 domínios com deficiência mantêm o risco em torno de $3 \%$, porém, dificuldades em 4 ou mais domínios eleva o risco de morte para $5,68 \%$.

Um instrumento contendo 40 variáveis foi desenvolvido com o objetivo de avaliar o potencial de uma rede neural artificial (ANN) ${ }^{39} \mathrm{em}$ predizer a sobrevida de idosos canadenses utilizando informações autorrelatadas. Os dados foram coletados a partir de 8.547 canadenses com idade entre 65 e 99 anos, acompanhados durante fevereiro de 1991 a 1997, no Canadian Study of Health and Aging (CSHA). A rede neural foi capaz de predizer taxa de sobrevida individual em aproximadamente 79,2\% dos casos em 10 simulações.

Outra tentativa de predizer o risco de morte e necessidade de institucionalização, foi a aplicação do CSHA Functional Scale (70 itens), por médicos clínicos que de acordo com o julgamento categoriza o escore individual dentre 7 estágios de "boa forma". Esta escala foi aplicada em 2.305 pessoas. Segundo os autores, o estudo teve boa correlação com prognóstico de fragilidade incluindo o risco de morte ou institucionalização ${ }^{40}$.

Em 1990, foi realizado um estudo utilizando o instrumento de rastreio Health Status Form $(\mathrm{HSF})^{41}$ para prever risco de fragilidade em idosos. O questionário autoadministrado contendo 8 páginas foi enviado por correspondência a 5.810 pessoas com idade igual ou superior a 65 anos, membros do Kaiser Permanent Northwest Region. Tempos depois, essas pessoas receberam uma carta para incentivar responder o questionário. O resultado do HSF foi comparado com formulários preenchidos por enfermeiras treinadas, com o sistema de controle de medicação e com registro de internação. Uma reavaliação por telefone ou visita domiciliar foi realizada a cada 90 dias. Os autores relatam que o sistema de controle de medicação reduz o consumo de fármacos entre os idosos.

$\mathrm{Na}$ Califórnia, EUA, foi aplicado um questionário com 11 questões, sobre atividades básicas e instrumentais de vida diária, para verificar se havia diferença de perda funcional quanto ao gênero. Concluíram que a lista é capaz de identificar a incapacidade nestas atividades ${ }^{42}$.

Gloth et al. ${ }^{43}$ desenvolveram e aplicaram o Frail Elderly Functional Assessment (FEFA) para ser aplicado a distância, de forma rápida e por profissional com pouco treinamento. Foram avaliadas 58 pessoas com mais de 65 anos que apresentavam resultado no MEEM $>18$, usuárias do centro geriátrico John Hopkins e outras três instituições urbanas de internação para reabilitação em Baltimore, EUA. Segundo os autores, o instrumento de 19 itens demonstrou ser confiável e sensível para detectar mudanças funcionais em idosos frágeis.

No Canadá, o Triage Risk Screening Tool $(\text { TRST })^{44}$, foi desenvolvido e aplicado em 2004 em 129 pessoas com 64 anos ou mais que procuraram um hospital de emergência. $\mathrm{O}$ instrumento foi aplicado por enfermeiras que receberam treinamento durante 4 meses. Segundo os autores, o instrumento não pôde ser usado como único preditor de retorno ao centro de emergência ou necessidade de institucionalização, no período de 30 e 120 dias. Porém, o rastreio possibilita uma diminuição do custo na emergência hospitalar, uma vez que um evento adverso pode ser detectado precocemente.

O Elderly at Risk Rating Scale (EARRS) ${ }^{45}$, foi desenvolvido e aplicado em 1990 em idosos com mais de 75 anos de um centro dia e em residentes da comunidade. $\mathrm{O}$ instrumento contém 20 questões abrangendo 7 áreas específicas: função sen- 
sorial, mobilidade, condição mental, condição física, continência, envolvimento social e uso de medicamentos. Foi aplicado por médicos e enfermeiros, utilizando cerca de 10 minutos para concluir o rastreio. No estudo, o risco de perda funcional aumentou de acordo com a idade e entre as mulheres.

Em Tapei, Taiwan, em 2007 foi realizada a validação da versão telefônica do Chinese-Canadian Study os Health and Aging Clinical Frailty Scale (CSHA-CFS TV) ${ }^{46}$. Este instrumento de 17 questões mede a perda de peso, o cansaço, o baixo gasto energético, a lentidão e a fraqueza. Foi aplicado por 2 auxiliares de pesquisa, através de contato telefônico com duração máxima de 3 minutos, em 67 pacientes ambulatoriais de um centro médico local. Os indivíduos com limitações em 3 ou mais áreas são considerados frágeis, entre 1-2, pré-frágeis e nenhuma deficiência, robustos. Assim que a fragilidade era identificada, uma entrevista médica foi agendada.

Em Marseille, França ${ }^{47}$ durante 2007 e 2008, outro questionário autoadministrado foi desenvolvido e enviado por correspondência, compreendendo critérios físicos (cálculo do índice de massa corporal e nível de atividade física) e psicossociais (perguntando pela satisfação com a força muscular e capacidade de resistência). Indivíduos apresentando 3 ou mais pontos eram considerados frágeis, 1-2 pré-frágeis e nenhum, robusto. Os autores descrevem que os idosos categorizados como frágeis no início da pesquisa tiveram taxa de mortalidade 6 vezes mais alta quando comparado com os indivíduos robustos, após 3 anos de acompanhamento. O instrumento não é descrito na publicação.

Pannil ${ }^{48}$ desenvolveu e validou um questionário autoadministrado para identificar perda funcional, baixa saúde física e baixos recursos socioeconômicos, que visa rastrear os indivíduos que necessitavam de assistência domiciliar, institucionalização ou somente uma avaliação geriátrica ampla. Foi realizado em 1985 em uma clinica geriátrica universitária em Milwalkee. O instrumento contendo 9 questões autorrelatadas foi enviado pelo correio a 80 idosos, e um resultado igual ou superior a 4 pontos foi associado com perda funcional moderada, pior recurso socioeconômico e saúde física fraca.

Foram selecionados quatro estudos referentes ao instrumento de rastreio Identification of Seniors at Risk (ISAR). Este instrumento consiste em 6 questões autoadministradas, com possibilidade de resposta dicotômica sim/não e ponto de corte em 2 pontos ${ }^{49}$.
As questões referem à necessidade de auxilio nas atividades diárias antes de buscar a emergência, se vai precisar de mais ajuda, o número de hospitalizações nos últimos seis meses, problemas de visão, memória e número de medicações tomadas diariamente.

Foi aplicado na Holanda em $2005^{50}$, onde se comparou o uso de três instrumentos, ISAR (Identification of screening tool), TRST(triage risk screening tool) e VIP (Variable Indicative of Placement Risk). Os dois primeiros utilizados para rastrear risco de perda funcional. O objetivo do estudo foi verificar a acurácia na predição da perda funcional em idosos hospitalizados.

Enfermeiras treinadas aplicaram os três instrumentos em 213 indivíduos com 65 anos ou mais que falavam o idioma holandês e procuraram o serviço de emergência em um hospital universitário no período de outubro a dezembro de 2005. Os participantes da pesquisa foram contatados por telefone 14,30 e 90 dias após alta hospitalar, por quatro enfermeiras treinadas, para avaliação do estado funcional.

Os autores do estudo concluíram que tanto o ISAR quanto o TRST são bons instrumentos de rastreio para predizer a perda funcional após alta hospitalar. Porém, o baixo valor preditivo positivo e alto valor de falsos positivos podem resultar em intervenções mal dimensionadas.

$\mathrm{Na}$ Itália ${ }^{51}$, o ISAR foi aplicado em 200 pessoas com idade igual ou superior a 65 anos, que procuraram o serviço de emergência em 2 hospitais na cidade de Ancona no período de Junho a Julho de 2006. O questionário foi aplicado por assistente treinado e os pacientes foram acompanhados durante 6 meses após a primeira ida à emergência.

Os autores concluíram que o ISAR é um preditor válido para morte e reinternação hospitalar. Quando utilizado o ponto de corte em 2 obtevese alta sensibilidade e em 3 , alta especificidade.

Um estudo de dados secundários realizado por Dendukuri et al. ${ }^{52}$ utilizou informações de dois estudos que utilizaram o ISAR. Um foi no desenvolvimento do instrumento, o outro foi um ensaio clinico randomizado para avaliar a eficácia da intervenção pela enfermagem em idosos de alto risco. Os autores verificaram a validade concorrente do instrumento para detecção de comprometimento funcional grave e depressão na visita hospitalar e validade preditiva para aumento de sintomas depressivos, uso frequente de centros de saúde da comunidade e hospitalização repetida.

Segundo os autores, apesar de não haver validação para uso extra-hospitalar, a escala ISAR 
tem potencial para ser aplicado no cuidado primário e em clínicas.

Os autores verificaram que a especificidade do ISAR com ponto de corte em 2 foi de $83 \%$ e a sensibilidade de $63 \%$ para detectar depressão. Para descobrir acréscimo nos sintomas depressivos, a sensibilidade foi de $73 \%$ e a especificidade de $57 \%$. Resultado semelhante foi encontrado para detectar o uso frequente de centros comunitários de saúde.

Os autores relatam que após a intervenção pela enfermagem, houve redução dos atendimentos de emergência pela metade. Este resultado foi encontrado no estudo realizado entre Setembro de 1998 a 1 de abril de $1999^{53} \mathrm{em}$ dois centros de atenção terciária de Montreal, Canadá. Neste estudo o ISAR foi aplicado por um pesquisador assistente em pessoas com idade igual ou superior a 65 anos.

Os pacientes com escore 2 ou mais foram convidados a participar do estudo. Um grupo randomizado de 178 pessoas foi indicado para avaliação geriátrica pelo serviço de enfermagem. O cuidador principal do idoso também foi avaliado pelo enfermeiro.

Após confirmação que o indivíduo precisava de maior acompanhamento, o mesmo era encaminhado para a unidade ambulatorial da região em que residia. Este contato era feito por telefone ou visita pré-agendada ao médico de família.

Comparado ao grupo controle $(\mathrm{n}=210)$, os idosos que receberam a intervenção da enfermeira, tiveram melhor desfecho, como menos perda da capacidade funcional e maior satisfação do usuário e do cuidador com a abordagem. Esta maior satisfação ocorreu principalmente entre os pacientes mais debilitados e os cuidadores do gênero feminino.

Em 1984, nos Estados Unidos, um grupo de pesquisadores ${ }^{54}$ definiu 8 critérios para rastrear idosos com risco de admissão hospitalar repetidamente no futuro. $\mathrm{O}$ instrumento foi aplicado em uma amostra probabilística da população norte americana civil, não institucionalizada, maior de 70 anos. Esta foi acompanhada durante 6 anos.

Os autores definiram repetidas internações como 2 ou mais internações por problemas agudos no período de 1985 a 1988. Os idosos foram questionados sobre características demográficas, socioeconômicas, médicas, funcionais e sobre o uso recente de serviços de saúde. O resultado foi categorizado em valores de probabilidade de repetidas admissões hospitalares (Pra), variando de 0,25 a 0,60 , onde quanto maior o resultado do
Pra, maior o risco de readmissão hospitalar no período de 4 anos.

Foi demonstrada validade preditiva positiva do instrumento para estimar o risco de admissão hospitalar repetida. À época, não houve intervenção nos idosos identificados com risco $(7,2 \%)$, porém os autores relataram que os idosos identificados com baixo risco morreram menos que aqueles com maior risco de internação no período de 1985-1988. É sugerido que o menor número de internações leve à melhora da saúde e resulte num menor gasto com serviços hospitalares.

O instrumento desenvolvido por Boult et al. ${ }^{54}$, foi aplicado em 360 pessoas com mais de 65 anos, usuários de um serviço de saúde no Rio de Janeiro, Brasil, com a finalidade de organizar o fluxo do atendimento no serviço de modo hierarqui$\mathrm{zado}^{10}$. Na ocasião foi identificado que $11 \%$ da população possuía risco moderado ou alto de internação hospitalar.

Guerra e Ramos-Cerqueira ${ }^{55}$, aplicaram o mesmo questionário em um centro de saúde escola (CSE) de Botucatu, Brasil, no período de 1 de Janeiro a 31 de Dezembro de 2000, com o objetivo de organizar o atendimento à pessoas idosas. Foram avaliadas 305 pessoas com mais de 65 anos, de ambos os sexos.

As autoras constataram que o risco de internação hospitalar foi elevado $(43,6 \%)$ considerando o somatório dos riscos médio, médio alto e alto. Por outro lado, muitos idosos classificados tendo baixo risco utilizaram várias consultas médicas no CSE, no ano anterior à pesquisa.

Outra experiência da utilização do questionário desenvolvido por Boult et al. ${ }^{54}$, realizado no Rio de Janeiro, foi sua aplicação no período de outubro a novembro de $2005^{56}$. O instrumento foi aplicado em 2637 usuários idosos ( $>65$ anos) de um plano de saúde através de contato telefônico de um operador e checado por uma enfermeira. O objetivo da aplicação do instrumento foi identificar os indivíduos com risco de internação.

As autoras relataram impasses no contato telefônico devido ligações não atendidas, linha telefônica ocupada ou com número errado, expondo a importância da atualização do cadastro dos usuários pelos serviços de saúde.

Na população avaliada $10,46 \%$ dos entrevistados apresentaram risco médio alto e alto para internação enquanto $76,03 \%$ dos usuários foram identificados com baixo risco de hospitalização.

A distribuição dos valores do Pra (probability of repetead admission) na população estuda- 
da também foi apresentada: Baixo $<0,30$, Médio 0,30- 0,39, Médio alto 0,400 - 0,499 e Alto 0,500.

Recentemente, o projeto PRO-AGE study (Prevention in Older people-Assessment in Generalists' practices), realizado em Londres (Inglaterra), Hamburgo (Alemanha) e Solothum (Suíça) aplicou um questionário contendo 34 páginas, contemplando 19 domínios da saúde, o HRA. Entre as questões apresentadas, foi calculado o Pra da população avaliada ${ }^{15,57}$.

Em Londres e Solothum o HRA foi aplicado em pessoas com idade igual ou superior a 65 anos, diferente de Hamburg onde a população avaliada tinha 60 anos ou mais ${ }^{15}$. $\mathrm{O}$ instrumento foi enviado por correspondência, juntamente com o questionário Pra e outros instrumentos de avaliação. Após devolução do questionário, parte do grupo recebeu intervenção que variou entre inclusão dos dados ao prontuário eletrônico e notificação do risco de saúde a equipe médica local (Londres), participação em grupo de intervenção em centro geriátrico ou visita de enfermeira treinada (Hamburg) e visita domiciliar por enfermeira treinada que realizava uma avaliação especializada quando necessário (Solothum).

Os primeiros resultados do Questionário Pra aplicado nos participantes do estudo PRO-AGE apontam um risco baixo de hospitalização nos grupos sob intervenção e de controle $(0,27 \mathrm{em}$ Londres, 0,30 em Hamburg e 0,29 em Solothun).

Em 2005, o Questionário Pra foi aplicado em 515 pessoas com mais de 60 anos, cadastradas no serviço de atenção básica de Progresso, Brasil ${ }^{58}$. A pesquisa objetivou verificar a validade do instrumento numa amostra da população idosa brasileira para prever risco de internação repetida.

Neste estudo, a estratificação de risco para hospitalização mostrou-se estatisticamente significativa na seguinte forma: risco baixo (Pra $<$ $0,30)$, risco médio $(\operatorname{Pra}=0,30-0,49)$ e risco alto $($ Pra $=0,500)$. Utilizando o ponto de corte em 0,4 , apresentou melhor efetividade para identificar idosos com alto risco de hospitalização em seis meses.

Os autores concluem que o uso do questionário Pra na rotina das equipes de saúde deve ser considerada, uma vez que possibilitará intervenções para evitar as hospitalizações.

\section{Discussão e conclusão}

Na revisão da literatura foram detectados cenários e experiências variadas quanto à aplicação de instrumentos de rastreio para risco de perda funcional, fragilidade ou admissão hospitalar, realizadas em diversos países.

A motivação parece ser um ponto comum na aplicação destes instrumentos. A necessidade de direcionar a assistência à saúde do idoso impulsionou o desenvolvimento de inúmeras ferramentas que rastreiem idosos com saúde vulnerável. Supõe-se que ao evitar a perda funcional, fragilidade e maior uso dos serviços de saúde aumenta-se a qualidade de vida dessas pessoas e se reduz os gastos em saúde.

Não houve consenso quanto à idade para se iniciar o rastreio de risco de fragilidade ou perda funcional, porém a maioria dos estudos excluiu idosos com perda cognitiva avançada, bem como os institucionalizados.

A aplicação dos instrumentos de rastreio foi realizada preferencialmente utilizando questionários curtos por meio postal ou entrevistas por profissional não médico, previamente treinado. $\mathrm{Na}$ maioria dos questionários autorreferidos os entrevistados puderam valer-se de auxílio familiar ou cuidador principal para preenchimento.

A reavaliação dos usuários foi realizada principalmente por contato telefônico ou visita domiciliar a cada 30 ou 90 dias.

O questionário Pra foi o único instrumento aplicado por telefone, pessoalmente e por correspondência. Foi aplicado para identificar idosos com risco de admissão hospitalar repetida, objetivo de seu constructo, mas também utilizando sua categorização para organizar a assistência à saúde de modo hierarquizado.

O ISAR, desenvolvido para prever o risco de perda funcional de idosos após alta hospitalar, também foi apontado como preditor válido para mortalidade e reinternação. Foi ainda aplicado por correspondência e teve sua utilização sugerida na atenção primária e em clínicas.

Nos estudos em que após a identificação do risco foi realizada alguma intervenção, os desfechos variaram de redução da perda funcional, número de admissão no setor de emergência, número de óbitos, redução de gastos com saúde e ainda uma maior satisfação do usuário com a assistência prestada.

As intervenções foram desenvolvidas em sua maioria por enfermeiras especialistas ou treinadas, sendo responsáveis por avaliar mais detalhadamente, orientar e acompanhar por determinado período o idoso, ou ainda encaminha-lo ao atendimento médico quando necessário.

Foi observado que a utilização dos instrumentos fora do país de origem não foi necessariamente precedida por validação transcultural, 
sendo assim, os resultados encontrados nestes trabalhos podem apresentar vieses culturais.

Entre as limitações deparadas durante a realização deste estudo, registrou-se a dificuldade de encontrar estudos de avaliação rigorosa da efetividade de intervenções, que atuassem em idosos sob risco de perda funcional e que avaliassem a efetividade da utilização do instrumento de rastreio na população.

Diante das conclusões e limitações apresentadas, observa-se a necessidade de trabalhos com melhor qualidade, considerando as características culturais, sociais e econômicas a partir da validação transcultural do instrumento escolhido. Além disso, é importante mais estudos de intervenção sobre o risco desses idosos, com a avaliação da efetividade da aplicação do instrumento. A existência desses estudos possibilitaria maior segurança na aplicabilidade de um método de rastreio de risco de perda funcional unificado para os usuários de um serviço de saúde ou, de todo um país.

\section{Colaboradores}

CP Caldas, RP Veras, LB Motta, KC Lima, CBS Kisse, CVM Trocado e ACLC Guerra participaram igualmente de todas as etapas de elaboração do artigo.

\section{Referências}

1. Wagner JT, Bachmann LM, Boult C, Harari D, von Renteln-Kruse W, Egger M, Beck JC, Stuck AE. Predicting the risk of hospital admission in older persons-validation of a brief self-administered questionnaire in three European countries. J Am Geriatr Soc 2006; 54(8):1271-1276.

2. Lourenço RA, Martins CSF, Sanchez MAS, Veras RP. Assistência ambulatorial geriátrica: hierarquização da demanda. Rev Saude Publica 2005; 39(2): 311-8.

3. Counsell SR, Callahan CM, Tu W, Stump TE, Arling GW. Cost Analysis of the Geriatric Resources for Assessment and Care of Elders Care Management Intervention. J Am Geriatr Soc 2009; 57(8):14201426.

4. Bielaszka-DuVernay C. The "GRACE" model: inhome assessments lead to better care for dual eligibles. Health Aff (Millwood) 2011; 30(3):431-434.

5. Góis ALB, Veras RP. Informações sobre a morbidade hospitalar em idosos nas internações do Sistema Único de Saúde do Brasil. Cien Saude Colet 2010; 15(6):2859-2869.

6. Taddeo OS, Gomes KWL, Caprara A, Gomes AMA, Oliveira GC, Moreira TMM Moreira. Acesso, prática educativa e empoderamento de pacientes com doenças crônicas. Cien Saude Colet 2012; 17(11):29232930.

7. Mitre SM, Andrade EIG, Cotta RMM. O acolhimento e as transformações na práxis da reabilitação: um estudo dos Centros de Referência em Reabilitação da Rede do Sistema Único de Saúde em Belo Horizonte, MG, Brasil. Cien Saude Colet 2013; 18(7):1893-1902. 
8. Kerber NPC, Kirchhof ALC, Cezar-Vaz MR. Bonding and home care satisfaction among elderly people. Texto \& Contexto Enferm 2008; 17(2):304-312.

9. Malta DC, Cecílio LCO, Merhy EH, Franco TB, Jorge AO, Costa MA. Perspectivas da regulação na saúde suplementar diante dos modelos assistenciais. Cien Saude Colet 2004; 9(2):433-444.

10. Veras R. Em busca de uma assistência adequada à saúde do idoso/ : revisão da literatura e aplicação de um instrumento de detecção precoce e de previsibilidade de agravos. Cad Saude Publica 2003; 19(3):705-715

11. Malta DC, Merhy EE. O percurso da linha do cuidado sob a perspectiva das doenças crônicas não transmissíveis. Interface (Botucatu) 2010; 14(34):593-605.

12. Pel-Littel RE, Schuurmans MJ, Emmelot-Vonk MH, Verhaar HJJ. Frailty: defining and measuring of a concept. J Nutr Health Aging 2009; 13(4):390-394.

13. McCusker J, Verdon J, Tousignant P, de Courval LP, Dendukuri N, Belzile E. Rapid emergency department intervention for older people reduces risk of functional decline: results of a multicenter randomized trial. J Am Geriatr Soc 2001; 49(10):1272-1281.

14. Walston J, Hadley EC, Ferrucci L, Guralnik JM, Newman AB, Studenski SA, Ershler WB, Harris T, Fried LP. Research agenda for frailty in older adults: toward a better understanding of physiology and etiology: summary from the American Geriatrics Society/National Institute on Aging Research Conference on Frailty in Older Adults. Health Aff (Millwood) 2006; 54(6):991-1001.

15. Stuck AE, Kharicha K, Dapp U, Anders J, von Renteln-Kruse W, Meier-Baumgartner HP, Iliffe S, Harari D, Bachmann MD, Egger M, Gillmann G, Beck JC, Swift CG. The PRO-AGE study: an international randomised controlled study of health risk appraisal for older persons based in general practice. BMC Med Res Methodol 2007; 7:2.

16. Pijpers E, Ferreira I, Stehouwer CD a, Nieuwenhuijzen Kruseman AC. The frailty dilemma. Review of the predictive accuracy of major frailty scores. Eur J Intern Med 2012; 23(2):118-123.

17. Lepeleire J De, Iliffe S, Mann E, Degryse JM. Frailty: an emerging concept for general practice. $\mathrm{Br} J$ Gen Pract 2009; 59(562):e177-e182.

18. Chan FH. Frailty in Older People. Medical Bulletin 2008; 11(5):11-13.

19. Poltawski L, Goodman C, Iliffe S, Manthorpe J, Gage H, Shah D, Drennan V. Frailty scales-their potential in interprofessional working with older people: a discussion paper. J Interprof Care 2011; 25(4):280-286.

20. Pialoux T, Goyard J, Lesourd B. Screening tools for frailty in primary health care: a systematic review. Geriatr Gerontol Int 2012; 12(2):189-197.

21. Gobbens RJJ, Assen MALM Van, Luijkx KG, Wijnen-sponselee MT, Schols JMGA. The Tilburg Frailty Indicator/: Psychometric Properties. J Am Med Dir Assoc 2010; 11(5):344-355.

22. Santiago LM, Luz LL, Mattos IE, Gobbens RJJ. Adaptação transcultural do instrumento Tilburg Frailty Indicator (TFI) para a população brasileira. Cad Saude Publica 2012; 28(9):1795-801.
23. Romero-ortuno R, Walsh CD, Lawlor BA, Kenny RA. A Frailty Instrument for primary care/ : findings from the Survey of Health, Ageing and Retirement in Europe (SHARE). BMC Geriatr 2010; 10(57): 1-12.

24. Mitnitski AB, Graham JE, Mogilner AJ, Rockwood K. Frailty, fitness and late-life mortality in relation to chronological and biological age. BMC Geriatr 2002; 8(2):1-8.

25. Amici A, Pecci MT, Linguanti A, Passador P, Ponzanetti A, De Angelis R, Martinelli V, Zaccone M, Marigliano V, Cacciafesta M. Self-administrated test based on the Marigliano-Cacciafesta Polypathological Scale (MCPS), as a screening tool for early identification of frailty in the elderly: a cohort study. Arch Gerontol Geriatr 2011; 52(1):e60-e65.

26. Moore AA, Siu AL. Screening for common proble$\mathrm{ms}$ in ambulatory elderly: clinical confirmation of a screening instrument. Am J Med 1996; 100(4):438443

27. Barber JH, Wallis JB, Mckeating E. A postal screening questionnaire in preventive geriatric care. $J R$ Coll Gen Pract 1980; 30(210):49-51.

28. Kempen GIJM, Miedema I, Ormel J, Molenar W. The Assessment of Disability With the Groninguen Activity Restriction Scale Conceptual Framework and Psycometric Properties. Soc Sci Med 1996; 43(11):1601-1610.

29. Hébert R, Bravo G, Korner-Bitensky N, Voyer L. Predictive validity of a postal questionnaire for screening community-dwelling elderly individuals at risk of functional decline. Age Ageing 1996; 25(2):159-167.

30. Walker L, Jamrozik K, Wingfield D. The Sherbrooke Questionnaire predicts use of emergency services. Age Ageing 2005; 34(3):233-237.

31. Daniels R, van Rossum E, Beurskens A, van den Heuvel W, de Witte L. The predictive validity of three self-report screening instruments for identifying frail older people in the community. $B M C$ Public Health 2012; 12:69.

32. Marín PP, Kornfeld REM. Medición del riesgo funcional en adultos mayores pobres, con un instrumento validado en Canadá. Revista Médica de Chile 1998; 126(11):1316-1322.

33. Raîche M, Hébert R, Dubois M-F. PRISMA-7: a case-finding tool to identify older adults with moderate to severe disabilities. Arch Gerontol Geriatr 2008; 47(1):9-18.

34. Hebert R, Raîche M, Dubois MF, Gueye N'Deye R, Dubuc N, Tousignant $M$, and THE PRISMA Group. Impact of PRISMA, a Coordination-Type Integrated Service Delivery System for Frail Older People in Quebec. J Gerontol: Soc Sci 2010; 65B(1):107-118.

35. Kerse N, Boyd M, McLean C, Koziol-McLain J, Robb G. The BRIGHT tool. Age Ageing 2008; 37(5): 553-588.

36. Maly RC, Hirsch SH, Reuben DB. The performance of simple instruments in detecting geriatric conditions and selecting community-dwelling older people for geriatric assessment. Age Ageing 1997; 26(3):223-231.

37. Matthews M, Lucas A, Boland R, Hirth V, Odenheimer G, Wieland D, Williams H, Eleazer GP. Use of a questionnaire to screen for frailty in the elderly: an exploratory study. Aging Clin Exp Res 2004; 16(1):34-40. 
38. Guilley E, Ghisletta P, Armi F, Berchtold A, d'Epinay CL, Michel J-P, Ribaupierre A. Dynamics of Frailty and ADL Dependence in a Five-Year Longitudinal Study of Octogenarians. Res Aging 2008; 30(3):299317.

39. Song X, Mitnitski A, MacKnight C, Rockwood K. Assessment of individual risk of death using selfreport data: an artificial neural network compared with a frailty index. J Am Geriatr Soc 2004; 52(7):11801184.

40. Rockwood K, Song X, Macknight C, Bergman H, Hogan DB, Mcdowell I, Mitnitski A. A global clinical measure of fitness and frailty in elderly people. CMAJ 2005; 173(5):489-495.

41. Brody KK, Johnson RE, Douglas Ried L. Evaluation of a self-report screening instrument to predict frailty outcomes in aging populations. Gerontologist 1997; 37(2):182-191.

42. Saliba D, Orlando M, Wenger NS, Hays RD, Rubenstein LZ. Identifying a short functional disability screen for older persons. J Gerontol A Biol Sci Med Sci 2000; 55(12):M750-M756.

43. Gloth FM, Scheve AA, Shah S, Ashton R, McKinney R. The Frail Elderly Functional Assessment questionnaire: its responsiveness and validity in alternative settings. Arch Phys Med Rehabil 1999; 80(12):1572-1576.

44. Fan J, Worster A, Fernandes CMB. Predictive validity of the triage risk screening tool for elderly patients in a Canadian emergency department. Am J Emerg Med 2006; 24(5):540-544.

45. Donald IP. Development of a modified Winchester disability scale-the elderly at risk rating scale. $J$ Epidemiol Community Health 1997; 51(5):558-563.

46. Chan D-CD, Tsou H-H, Chen C-Y, Chen C-Y. Validation of the Chinese-Canadian study of health and aging clinical frailty scale (CSHA-CFS) telephone version. Arch Gerontol Geriatr 2010; 50(3):e74-e80.

47. Barreto PDS, Greig C, Ferrandez A-M. Detecting and categorizing frailty status in older adults using a self-report screening instrument. Arch Gerontol Geriatr 2012; 54(3):e249-e254.

48. Pannill FC. A patient-completed screening instrument for functional disability in the elderly. Am J Med 1991; 90(3):320-327.

49. McCusker J, Bellavance F, Cardin S, Trepanier S, Verdon J, Ardman M. Detection of older people at increased risk of adverse health outcomes after an emergency visit: The ISAR Screening Tool. $J$ Am Geriatr Soc 1999; 47(10):1229-1237.

50. Braes T, Flamaing J, Sterckx W, Lipkens P, Sabbe M, de Rooij SE, Schuurmans MJ, Moons P, Milisen $\mathrm{K}$. Predicting the risk of functional decline in older patients admitted to the hospital: a comparison of three screening instruments. Age Ageing 2009; 38(5):600-603.
51. Salvi F, Morichi V, Grilli A, Lancioni L, Spazzafumo L, Polonara S, Abbatecola AM, De Tommaso G, Dessi-Fulgheri P, Lattanzio F. Screening for frailty in elderly emergency department patients by using the Identification of Seniors At Risk (ISAR). J Nutr Health Aging 2012; 16(4):313-318.

52. Dendukuri N, McCusker J, Belzile E. The identification of seniors at risk screening tool: further evidence of concurrent and predictive validity. $J \mathrm{Am}$ Geriatr Soc 2004; 52(2):290-296.

53. McCusker J, Verdon J, Tousignant P, de Courval LP, Dendukuri N, Belzile E. Rapid emergency department intervention for older people reduces risk of functional decline: results of a multicenter randomized trial. J Am Geriatr Soc 2001; 49(10):1272-1281.

54. Boult C, Dowd B, McCaffrey D, Boult L, Hernandez R, Krulewitch H. Screening Elders for Risk of Hospital Admission. J Am Geriatr Soc 1993; 41(8):811-881.

55. Guerra IC, Ramos-Cerqueira ATA. Risco de hospitalizações repetidas em idosos usuários de um centro de saúde escola Risk of repeated hospitalizations in elderly users of an academic health center. Cad Saude Publica 2007; 23(3):585-592.

56. Estrella K, Oliveira CEF, Sant'Anna AA, Caldas CP. Detecção do risco para internação hospitalar em população idosa: um estudo a partir da porta de entrada no sistema de saúde suplementar. Cad Saude Publica 2009; 25(3):507-512.

57. Stuck AE, Kharicha K, Dapp U, Anders J, von Renteln-Kruse W, Meier-Baumgartner HP, Harari D, Swift CG, Ivanova K, Egger M, Gillmann G, Higa J, Beck JC, Iliffe S. Development, feasibility and performance of a health risk appraisal questionnaire for older persons. BMC Med Res Methodol 2007; 7:1.

58. Dutra MM, Moriguchi EH, Lampert MA, Poli-deFigueiredo CE. Validade Preditiva de instrumento para identificação do idoso em risco de hospitalização. Rev Saude Publica 2011; 45(1):106-112.

Artigo apresentado em 15/03/2013

Aprovado em 19/03/2013

Versão final apresentada em 20/03/2013 